\title{
Mothers' Breastfeeding Experiences in the NICU
}

\author{
Camille A. Boucher, RN, MSc(A) \\ Paola M. Brazal, RN, MS $S_{\mathcal{c}}(A)$ \\ Cynthia Graham-Certosini, RN, MSc(A) \\ Kathryn Carnaghan-Sherrard, $R N, M S_{c}(A)$ \\ Nancy Feeley, RN, PhD
}

L

ITTLE IS KNOWN ABOUT HOW MOTHERS PERCEIVE their breastfeeding experiences in the NICU. The purpose of this study was to explore the emotional and physical experiences mothers undergo when they are able to place their infant to their breast with the intent of breastfeeding. Mothers described their experiences in terms of maintaining milk production, the regimen of the NICU, mother as learner, personal motivation, and forming attachments.

Mothers emphasized the role breastfeeding played in allowing their infants to recognize them. They believed that returning home with their infant would facilitate the breastfeeding experience. Findings from this study can help nurses enhance the important role they play in mothers' NICU breastfeeding experiences.

\section{LITERATURE REVIEW}

A large body of literature supports the many beneficial properties of human milk for infants, such that the World Health Organization and United Nations International Children's Emergency Fund have as their mandate the promotion of breastfeeding. Research further endorses the clinical practice of using human milk for feeding preterm (born at $<37$ weeks gestation) and low birth weight infants $(<2,500 \mathrm{~g})$ because it can provide nutrients not found in formula. ${ }^{1}$ Human milk provides preterm infants with nutritional, gastrointestinal, immunologic, developmental, and psychological advantages. ${ }^{2}$ It is not uncommon for parents, especially mothers, to feel disconnected from their parental role while their infant is in the NICU. Because they are not involved in their infant's care, mothers in the NICU may feel as though their child belongs to the nurses and other caregivers. ${ }^{3}$ Breastfeeding or supplying human milk for her infant can provide the mother with a vital role in his care. ${ }^{4}$ Mothers consider breastfeeding to be one of the few ways they can fulfill their maternal role. Breastfeeding is therefore believed to be closely associated

\section{Disclosure}

The authors disclose no relevant financial interests or affiliations with any commercial interests. 
with a mother's idea of "good mothering" because only she can offer her infant this particular type of nurturing. ${ }^{5}$

Although evidence demonstrates a strong link between breastfeeding and many physical and psychological health benefits for both mothers and their infants, breastfeeding rates in the U.S. and in Canada are less than desired, particularly long term. Among mothers of healthy infants in the U.S., 74 percent initiated breastfeeding shortly after birth in $2006 .{ }^{6}$ In Canada, 90.3 percent of infants are breastfeeding in the early postpartum period according to a recent study. ${ }^{7}$ Exclusive breastfeeding, however, quickly declines to 13.6 percent in the U.S. and 14.4 percent in Canada by six months postpartum. ${ }^{7,8}$

Data on breastfeeding rates for preterm infants are limited. Although mothers of preterm infants are less likely to initiate and maintain breastfeeding than mothers of healthy term infants, data suggest that breastfeeding initiation rates for preterm infants are promising, whereas breastfeeding continuation is suboptimal. ${ }^{9,10}$ Bernaix and colleagues have found that mothers are faced with many barriers to breastfeeding a preterm infant, such as the stressful nature of the NICU, the physical separation from their infant, fatigue and anxiety associated with having an ill infant, the lack of privacy on the NICU, limited milk supply, and conflicting and inaccurate knowledge concerning the importance of human milk for their infants. ${ }^{4}$ These barriers may influence mothers' breastfeeding initiation and progression rates.

A study by Flacking and colleagues explored mothers' breastfeeding experiences during their infant's stay in the NICU, as well as after discharge. The findings suggest that mothers perceived breastfeeding to be an indicator of their infant's vitality and strength. Mothers also expressed that breastfeeding provided them with a feeling of importance and normality. ${ }^{11}$ Although this retrospective study provided interesting data, it does not necessarily accurately represent mothers' breastfeeding experiences while in the NICU because at the time of the interviews, all the mothers were successfully feeding their infants at the breast and their infants were no longer in the NICU.

In summary, much of the literature that examines the breastfeeding of preterm infants or those admitted to the NICU focuses on the nutritional, immunologic, and physiologic importance of human milk. Much less is known about mothers' perceptions of their own breastfeeding experiences while in the NICU. The mother's experience of providing human milk for her infant and the struggles involved in establishing breastfeeding in an NICU setting may play an important role in breastfeeding outcomes for this population. Consequently, it is imperative to acquire an understanding of the mother's experiences as she is immersed in this process. The purpose of this study, therefore, was to explore the maternal experience of breastfeeding initiation and progression in the NICU, which is a highly medical, technical, and unfamiliar setting.
Literature concerning breastfeeding of preterm infants is often unclear about the definition of breastfeeding. The term has been used interchangeably to refer to infants receiving only human milk directly from the mother's breast and to those receiving human milk via other means such as bottle or enteral feeding. For the purpose of this study, breastfeeding was defined as the skin-to-skin contact that occurs when an infant's face touches the mother's breast with the intention of having the infant suckle the nipple to obtain human milk. This study describes and documents this experience from the mother's perspective. Knowledge accrued in this qualitative study will assist nurses to better understand the maternal experience of breastfeeding in an NICU setting. This knowledge can be used to enhance support of mothers in the NICU and to help them attain their breastfeeding goals.

\section{METHODS}

A qualitative descriptive design was chosen for this study. ${ }^{12,13}$ Basic qualitative descriptive studies provide a summary of an event or experience in the everyday language of the event, with a low level of inference by the researcher. ${ }^{12}$ This design is useful in providing answers to clinically relevant questions and was considered the method of choice to describe mothers' breastfeeding experiences in the NICU.

A convenience sample of ten mothers was recruited from a Level III NICU in a large urban hospital. The sample included mothers whose infants were between 33 and 36 weeks gestational age at the time of the study. Only mothers who had begun to breastfeed and who were able to understand and speak either English or French were included. Infants had to have been in the NICU for at least five days, because this was deemed sufficient time to allow mothers to initiate breastfeeding and receive any lactation support and resources offered by the NICU staff. The sample excluded families with infants who had been apprehended. It also excluded mothers with a history of substance abuse and those who were undergoing methadone treatment. Lastly, it excluded mothers of infants with a diagnosed Grade III or IV cerebral hemorrhage, those with infants who were being ventilated, and mothers of infants with congenital abnormalities that might interfere with breastfeeding.

Five of the participants were primiparas; five mothers were multiparas. Three mothers had previous breastfeeding experience ranging from six months to one year. Maternal age ranged from 24 to 35 years, and all mothers except one had an involved partner. Seven mothers had obtained postsecondary education, and three had completed high school only. Infants were born between 27 and 34 weeks gestation and weighed between 740 and $1,970 \mathrm{~g}$ at birth. At the time of interview, infants had been in the NICU from two to seven weeks and were between 33 and 36 weeks gestational age.

Ethical approval from the institution's Research Ethics Committee was obtained. Potential participants were identified and approached by the NICU assistant head nurse. Interested mothers were referred to the researchers, who 
explained the purpose and procedures of the study in more detail. If a mother agreed to participate, a signed informed consent was obtained, and an interview was scheduled at a time convenient for the participant.

Data were collected through face-to-face semistructured interviews, which were audiotaped. Main guiding, openended questions were used to explore and encourage mothers to provide in-depth details about their breastfeeding experience. The following questions guided the interviews:

1. Can you tell me about your experience of putting your baby to your breast?

2. So far, has your breastfeeding experience been what you expected?

The first two authors, who were not involved in the infants' care, conducted the interviews. Interviews were conducted on-site in the NICU family room to ensure privacy and were approximately one hour in duration. Participants were referred to the clinical nurse specialist if they had any questions regarding breastfeeding or infant care.

Immediately following all interviews, the interviewer made notes detailing the participant's nonverbal behavior and any relevant contextual information. Audiotapes were transcribed verbatim, and accompanying observations were added to the transcripts. Qualitative content analysis was the approach used to analyze the data. Analysis of the data was conducted concurrently with data collection. Transcripts were first read through. Upon rereading, categories were created by grouping similar ideas generated from the data collected during the interviews. This list of categories was further examined to remove repetition or duplication. As data accumulated, they were compared and contrasted to previously collected data. Categories were reinforced or changed to better reflect the data. When data collection was finished, a final list of categories was produced.

To ensure validity, researchers independently analyzed and interpreted data before coming to a consensus on the final list of categories. To establish credibility, feedback regarding emerging data and interpretations were provided to participants throughout the interview process to ensure that the researchers had a reasonable understanding of their experiences. Peer debriefing sessions were held with both researchers and expert NICU clinicians to further ensure credibility. In these debriefing sessions, the interpretation of the data and the emerging categories were discussed to ensure that the categories were appropriate and best represented the data.

\section{RESULTS}

Breastfeeding was a decision the mothers had made prior to or during their pregnancy. Following the birth of their infant, mothers quickly recognized that their breastfeeding experience would be different from what they had anticipated. Breastfeeding an infant in the NICU added new dimensions to the act of breastfeeding and to the breastfeeding experience itself. Mothers described their experiences in terms of maintaining milk production, the regimen of the
NICU, mother as learner, personal motivation, and forming attachments.

\section{Maintaining Milk Production}

When mothers described their breastfeeding experience, a predominant theme that emerged was their ability to sustain milk production to adequately supply the needs of their infant. Although all mothers had sufficient milk at the time of their interviews, many worried that their milk production would diminish and not meet the increasing demands of their infants as they grew. Others were concerned that they would eventually run out of milk, especially if their infants did not take the breast exclusively. Mothers explained that having to continue to mechanically express their milk would ultimately diminish their production because of the exhausting nature of the process and the less efficient milk retrieval. "I hope to put him to the breast because having to continue to pump my milk is tiring and my milk quantity will begin to diminish. This is stressful."

One mother experienced a brief loss of milk and was not able to put her infant to her breast. Not being able to breastfeed her infant led to feelings of failure. Other mothers agreed that they too would feel that they had failed if they could not breastfeed because of insufficient milk production and supply: "When I had no milk, that was a terrible feeling... I felt like a failure. I'm not going to be able to feed my child... something that seemed so natural, so inherent. So why am I not producing milk?"

Because of their worries surrounding milk production, mothers dedicated a considerable amount of time and energy to executing strategies that would maintain their milk supply. Mechanically expressing their milk was the predominant strategy used by all mothers to ensure that their milk production continued when they were not exclusively breastfeeding. Getting adequate rest and striving for a healthy diet were other strategies that mothers used to maintain an adequate milk supply. "Sleep has a huge impact on my milk. If I have a nap the day before, the next day I have great milk. If I have no nap, I have much less milk." Another mother commented, "I want breast milk, so I'm trying to nap. I'm very conscious of the food that I eat so that she gets the benefits of all of it."

\section{Regimen of the NICU}

Many mothers described their breastfeeding experience in the NICU as being very structured because the infant's feeding schedule was not necessarily based on individual infant needs, but rather on the unit's structural organization and timetable. Breastfeeding took place at very precise hours, and the amount of time infants were given to attempt to breastfeed was also limited. Mothers felt that this strict regimen did not fit with their infant's actual feeding patterns. According to one mother, "[Nurses] feed [the babies] every three hours here, but it's not necessarily the pattern of the child's hunger. So, there's certain feedings where all she 
wants to do is sleep." If the infants have to be wakened for a scheduled feeding, they may not necessarily be hungry and may be too tired to breastfeed, thereby falling asleep at the breast.

Contrary to the regimented breastfeeding sessions in place in the NICU, mothers expressed that breastfeeding should be provided on an on-demand basis. One mother said, "[Medical staff] can't let him be a baby, be himself... If he was truly hungry, he would end up taking my breast." Some mothers also felt that their infants might consume more milk if they were given more time at the breast. One mother stated, "[N]urses don't want him to get exhausted either, so we limit [breastfeeding] to no more than 20 minutes. I think that if I fed him continually up to 40 minutes, he would get substantially a lot more." Mothers believed that the structured feeding routine implemented in the NICU was not conducive to their breastfeeding attempts.

\section{Mother as Learner}

This was the first experience with breastfeeding for seven of the ten mothers. The three mothers who did have breastfeeding experience with a term infant admitted that although there were similarities, there were many different aspects to breastfeeding a preterm infant. All mothers expressed the need not only to learn the necessary breastfeeding techniques, but also to learn to understand their infant's cues and behaviors in order to optimize the breastfeeding experience. To put their infants to the breast, mothers first needed to feel comfortable with holding them. All mothers were initially hesitant to hold their infants because of their size and delicateness. As one mother stated, "You feel like they're so fragile 'cause they're so small. So you don't feel comfortable." With time, mothers became more comfortable and confident in holding their infants, consequently feeling more at ease with breastfeeding. The mothers described the nurses as an important resource for them during this learning process. Mothers stated that nurses provided information and taught them techniques that promoted effective breastfeeding. Mothers were shown different positions and holds, how to manipulate their breast, and how to assess for a proper latch. One mother stated, "It's all about holding your breast; it's really the techniques. You need to know [your breast]. And you have to feel comfortable with it." One mother explained, "With breastfeeding, I always have a nurse with me still, 'cause they'll see little subtle things. They start you with a piece of information and then you try it and then they give you another piece of information.... I really need buckets of reinforcement from them and feedback." Mothers needed to be reassured about their ability to breastfeed effectively. Nurses also played a pivotal role in providing mothers with positive reinforcement surrounding their attempts to breastfeed. "[Nurses] can tell me that I'm doing well. I need a lot of reinforcement from the nurses. I need the nurses to tell me that I've positioned her neck the right way so that she's breathing."
Mothers quickly realized that breastfeeding was not an inherent skill but, rather, something that needed to be learned. For one mother, for example, an element of learning how to breastfeed successfully was to become an expert in observing and interpreting her infant's behavior. "You've got to learn how to read your baby... know exactly how much milk she's getting, learn if she's eating effectively, if she's latching properly.... I thought that everything with motherhood clicks instinctively. It doesn't."

Learning to breastfeed a preterm infant also meant that the mother had to become more in tune with her body and learn what she needed to do to maximize milk production. Mothers described that they needed to discover their own physical limitations and develop an understanding of their body's ability to function under various circumstances such as exhaustion, stress, and unsettling conditions. "To me the best thing to ensure effective breastfeeding is to know how your body works and what schedule works best, know your own system, your own schedule... and where's your sleep at.”

\section{Personal Motivation}

Mothers voiced several reasons that prompted their intention to breastfeed, and they had every intention of persevering and obtaining their goal of exclusive breastfeeding. Mothers repeatedly listed numerous physical advantages of human milk for the infant, such as nutritional benefits, immunity, and growth. Having preterm infants further strengthened their motivation to provide their infants with these benefits. Many mothers noted that they would have discontinued their attempts to breastfeed if their infants had not been preterm. One mother explained, "If breastfeeding had been this complicated and she had been full term, I would have quit, but since she's preterm, we push."

Mothers were motivated to breastfeed in the NICU because they believed their breastfeeding experience would be very different once their infants were discharged. Mothers felt that if they continued to breastfeed while they were in the NICU, they would eventually be able to reach their goal of breastfeeding exclusively when they returned home with their infants. One mother said, "I know once we get home, he is going to predominantly breastfeed." According to many mothers, having their infants at home would facilitate breastfeeding and help with their milk production. One mother stated, "[W] hen you go home and try to breastfeed, you're not going to be stressed, you're not going to be as tired because of traveling to the hospital, and you have your baby at home...." Another mother expressed, "I'm sure [breastfeeding] will be completely different once she's home. I'll breastfeed when she's hungry, and I'll care for her the way I know how..."

Although all mothers aspired to put their infants to the breast successfully, for some mothers the desire for their infants' more rapid weight gain and subsequent discharge took precedence over their preferred feeding method. Infants 
were often not getting enough milk solely from the breast. As a result, some mothers chose the feeding method that was most trouble free and that would lead to weight gain, usually bottle feeding, over breastfeeding. Many mothers voiced that this was a difficult decision to make: "[I]f I want to leave this place [NICU], he needs to be able to take the breast or the bottle, so... I always hesitate. Do I give him a bottle or do I try to put him at the breast?"

\section{Forming Attachments}

Mothers felt limited in the role they could play in the care of their infants in the NICU. They felt that the NICU environment limited their ability to develop a relationship with their infants. Mothers saw breastfeeding as an opportunity to hold and connect with their infants. One mother shared, " $[\mathrm{M}] \mathrm{y}$ pregnancy was interrupted early. I'm here now at the NICU. There's not much bonding time, so I think [breastfeeding] is the best way for my daughter and I to bond."

Breastfeeding was also seen as a way of continuing the connection that the mother had had with her infant when the baby was still in utero. According to one mother, "[Breastfeeding] is as close as I can get her to in utero." Another mother elaborated, saying, "[Breastfeeding] gives you the chance to continue... that link.... [I]t's as if you're still connected to her, as if you're pregnant." A few mothers saw breastfeeding as a way to compensate for what they perceived as an interrupted pregnancy. They felt breastfeeding was the best thing they could provide for their infants as a counterbalance to not being able to carry them to term. One mother disclosed, "[S]ince I couldn't keep her to full term, [breastfeeding] is the most I can do." According to another mother, "You really want to give him the best. Especially since I wasn't able to give him my uterus for forty weeks. I can at least give him my milk." Mothers felt involved with and connected to their infants when they were able to offer nourishment and contribute to their growth.

Mothers described breastfeeding as a way for their infants to recognize them, thereby helping in forming an attachment. According to one mother, "It's really the link, especially a preterm baby. You want him to recognize his mother, her odor... I always feared he'd forget me. When he takes the breast, he'll eventually make the connection. I know he won't forget me."

\section{DISCUSSION}

This study investigated the breastfeeding experience of mothers in the NICU. Mothers expressed concern with maintaining their milk production, and they felt that the NICU feeding regimen was not conducive to promoting their breastfeeding. Although breastfeeding was a challenge that required learning, it provided mothers with a sense of belonging, purpose, and worth. Consequently, mothers were highly motivated to maintain milk production and work within the confines of the regimen of the NICU in order to breastfeed their infants. Mothers were also motivated to continue their efforts to breastfeed in the NICU in anticipation of an easier breastfeeding experience at home. Nurses played a strong guiding, educational, and motivating role in providing mothers with the information and support they needed for a positive and rewarding breastfeeding experience. The study found that mothers placed great importance on the role breastfeeding plays in fostering mother-infant attachment.

One central aspect of the experience of breastfeeding in the NICU was the mother's concern regarding maintaining her milk supply. Although all of the women reported that they currently had sufficient milk for their infants, they worried that supply might become problematic for a variety of reasons. This concern is also very common among mothers of newborn infants not hospitalized in the NICU. An integrative review of the literature found that a large percentage of women report insufficient milk supply as the most common concern they have about breastfeeding and that 35-44 percent of women cite it as the primary reason for early cessation of breastfeeding. ${ }^{14} \mathrm{~A}$ relationship has been found between mothers' perceptions of inadequate milk supply and lack of confidence in their ability to parent. ${ }^{15}$ Supporting the maternal decision to breastfeed and reassuring the mother of her body's ability to produce milk can develop confidence. Furthermore, teaching mothers to read their infants' cues of hunger and fullness can allow them to feel more competent in their ability to breastfeed. ${ }^{15}$ Nurses must inform mothers of the importance of early and frequent mechanical expression of their milk postpartum for optimal milk production. ${ }^{16}$ In addition, promoting use of the electric breast pump at the infant's bedside promotes stimulation and emphasizes to the mother the important role she plays in providing her infant with human milk. ${ }^{17}$ Nurses are in an ideal position to discuss with mothers their milk expression experience. By demonstrating interest and providing encouragement, nurses give mothers the opportunity to ask questions and voice any concerns they may have.

Another prominent theme among mothers was the perception that the regimen followed for infant feeding in the NICU had negative consequences for their ability to breastfeed their infants. Infants in the NICU are often on a feeding schedule that consists of a set amount of milk and calculated feeding times to ensure that daily requirements are met. This practice may leave mothers with the impression that their beliefs and practices are of less importance than those of the NICU staff. Because mothers fed their infants in this NICU at a scheduled time rather than on demand, they may have planned their hospital visits around the NICU feeding regime. It is therefore important to involve mothers in their infants' feeding plans and to communicate any changes in their infants' schedules so that mothers can maximize their opportunities to breastfeed.

Mothers perceived themselves as learners while attempting to breastfeed and to interpret their infants' cues. Mothers' breastfeeding experiences required them not only to learn 
about their infants, but also to learn about themselves to optimize their breastfeeding experience. They needed to learn breastfeeding techniques and positions, as well as infant cues and behavior during feeding. They also needed to learn about their own bodies and how to optimize their milk production. Most (seven of ten) of the mothers did not have previous breastfeeding experience. They also reported that nurses played an integral role in helping them learn breastfeeding techniques and establish a certain level of comfort when holding their infants. Encouragement and assistance with breastfeeding from the nursing staff allowed mothers to come to know and understand their infants during breastfeeding. Nurses need to provide information, encouragement, and positive reinforcement and to teach various breastfeeding techniques to facilitate mothers' breastfeeding efforts.

The findings also revealed that the mothers were highly motivated to breastfeed their infants despite the challenges of doing so in the NICU. Mothers reported that human milk was the only thing they could give their infants while in the NICU. Numerous studies have found that the most stressful aspect of the NICU for the mother is the limitation of her role in her infant's care. ${ }^{18,19}$ Mothers of infants in the NICU may be unable to perform important tasks such as holding and caring for their infants. The emphasis that these mothers placed on the importance of breastfeeding may stem from their need to reclaim their maternal role in the context of their infants' NICU care, as well as providing them with an opportunity to enhance the process of maternal-infant attachment. This finding is consistent with a study by Lupton and Fenwick that found that mothers search for chances to interact physically with their infants and that they especially value providing human milk to them because it is something only they can do. ${ }^{5}$ Findings from the present study suggest that breastfeeding not only provides a physical connection between mother and infant, but also allows mothers to feel involved in their infants' care and to contribute to their growth and well-being.

Finally, this study found that another significant motivator for mothers was their belief that their breastfeeding experience would become less difficult and more pleasurable once they were home with their infants. Mothers anticipated that breastfeeding would be less challenging because of the less stressful home atmosphere, more opportunities for rest, a more flexible feeding schedule, and having their infants nearby. As Flacking and colleagues noted, in the home, mothers believe that they will be able to act in accordance with their beliefs and practices regarding their infants' care and that this will facilitate their ability to breastfeed successfully at home. ${ }^{20}$ The hope that breastfeeding will be easier once home may not only be a motivating factor to persist with their breastfeeding attempts while in the NICU, but it may also represent a way for these mothers to cope with the challenges they experience in the NICU.

Unfortunately, mothers' expectations about their breastfeeding experiences after discharge may not correspond with the realities of breastfeeding a preterm infant at home. A study by Callen and colleagues has found that there is a steady decline in mothers who continue to breastfeed following their infants' discharge from the hospital. ${ }^{21}$ Challenges that mothers face include emotional and physical fatigue as well as a sleepy infant who is difficult to arouse for feedings. ${ }^{9}$ Thus, an important component of breastfeeding education in the NICU is to provide mothers with information that may promote more realistic expectations regarding their milk production, as well as the breastfeeding challenges they may encounter when they return home with their infants. This may help mothers better understand and interpret their current breastfeeding experiences in the NICU and allow them to realistically anticipate their future breastfeeding experiences.

Breastfeeding support and education should not be limited to the infant's NICU stay. It should continue to be provided to mothers following discharge home. Mothers will likely face breastfeeding challenges when their infants come home, despite their belief that their experience will become easier following discharge. Prior to discharge, mothers' support systems should be assessed to ensure that they will be encouraged in their belief that they can breastfeed. Formal support systems, such as pediatricians or family doctors, as well as informal support systems, including family and friends, can help optimize the likelihood of mothers' achieving their anticipated breastfeeding experiences in the NICU as well as after discharge.

Other resources that can be integrated into mothers' support programs are role models. Nurses should facilitate and encourage opportunities for mothers to spend time with other mothers who have been through similar experiences in the NICU and have made the transition to a life outside the hospital. Supporting the mother in her breastfeeding efforts and reinforcing her ability to breastfeed may not only encourage her to breastfeed during the infant's hospital stay, but may also facilitate breastfeeding efforts at home.

Exploring mothers' perceptions of their breastfeeding experiences in the NICU made it apparent that breastfeeding was not merely a practical and technical task. The findings suggest that breastfeeding may make an important contribution to the fundamental process of maternal-infant attachment. Mothers perceived their breastfeeding experience to be an extension of their pregnancy. They believed that the mother-infant attachment process, which began well before birth, would continue to develop if they were able to breastfeed their infants successfully while in the NICU. Indeed, a woman's transition to motherhood begins during pregnancy as she starts to care for herself and her unborn child. ${ }^{22}$ The study participants also viewed breastfeeding as a way to optimize their infants' health and well-being, in light of the preterm birth.

The participants in this study described breastfeeding as a method for forming an attachment with their infants. They believed that breastfeeding could promote their infants' 
attachment to them by ensuring that their infants would recognize them, not only as mother, but also as the person who would nourish them. Some mothers feared that their infants would not recognize them or would forget them while in the NICU. This fear may have been prompted by the separation of mother and infant, mothers' limited participation in their infants' care, or their inability to be constantly present in the NICU. Although studies have found that mothers of term infants are confident that their infants will recognize them, mothers of preterm infants are not as sure. Maternal recognition by a preterm infant is vital to a woman's sense of self as a mother. ${ }^{23}$ Mothers reported that breastfeeding would enable their infants to distinguish them from other caregivers, such as nurses, who are integral players in the infants' survival and in fulfilling nutritional needs. Indeed, one study found that putting the infant to his mother's breast provides an opportunity to develop the ability to recognize the mother's unique milk odors. ${ }^{24}$ Nurses must recognize the importance mothers place on breastfeeding as a way of developing a relationship with their infants. They should also facilitate other types of maternal involvement in the infant's care to the greatest extent possible.

One limitation of this study is that the sample was quite heterogeneous with respect to infant gestational age at birth (27-34 weeks), and this might affect mothers' breastfeeding experiences. The study included mothers both with and without previous breastfeeding experience, and previous experience may influence the NICU breastfeeding experience. Further, the sample was limited to English- and French-speaking mothers, restricting the inclusion of ethnic minorities. It is unclear how culture may influence the experience of breastfeeding in the NICU. Mothers who are able to initiate breastfeeding in the NICU may be particularly motivated to do so and not typical. Yet another limitation of the study is that mothers were interviewed only once. The maternal experience of breastfeeding may evolve during infant hospitalization.

Several directions for future research arise from this study's findings. First, the breastfeeding experience of NICU mothers with and without previous breastfeeding experience could be compared. It would also be informative to examine the experience of mothers over time during their infants' NICU hospitalization. Future studies with a more diverse cultural representation are also needed. Although several studies have examined breastfeeding outcomes following discharge from the NICU, studies exploring mothers' expectations concerning their postdischarge breastfeeding experiences are sparse. The scarceness of literature comparing mothers' breastfeeding experiences in the NICU to their experiences after their infants' NICU discharge indicates the need for further research in this area.

\section{REFERENCES}

1. Gartner, L. M., Morton, J., Lawrence, R. A., Naylor, A. J., O’Hare, D., Schanler, R. J., \& Eidelman, A. I. American Academy of Pediatrics Section on Breastfeeding. (2005). Breastfeeding and the use of human milk. Pediatrics, 115, 496-506. doi:10.1542/peds.2004-2491

2. Newton, E. R. (2004). Breastmilk: The gold standard. Clinical Obstetrics and Gynecology, 47, 632-642. doi:10.1097/01.grf.0000136184.19927.98

3. Wigert, H., Johansson, R., Berg, M., \& Hellström, A. L. (2006). Mothers' experiences of having their newborn child in a neonatal intensive care unit. Scandinavian Journal of Caring Sciences, 20, 35-41. doi:10.1111/ j.1471-6712.2006.00377.x

4. Bernaix, L. W., Schmidt, C. A., Jamerson, P. A., Seiter, L. B., \& Smith, J. M. (2006). The NICU experience of lactation and its relationship to family management style. American Journal of Maternal Child Nursing, $31,95-100$.

5. Lupton, D., \& Fenwick, J. (2001). "They've forgotten that I'm the mum": Constructing and practicing motherhood in special care nurseries. Social Science o Medicine, 53, 1011-1021.

6. Centers for Disease Control and Prevention. (2010). Breastfeeding among U.S. children born 1999-2007. CDC National Immunization Survey. Retrieved from http://www.cdc.gov/breastfeeding/data/NIS_data/ index.htm

7. Chalmers, B., Levitt, C., Heaman, M., O'Brien, B., Sauve, R., \& Kaczorowski, J. (2009). Breastfeeding rates and hospital breastfeeding practices in Canada: A national survey of women. Birth, 36, 122-132. doi:10.1111/j.1523-536X.2009.00309.x

8. Centers for Disease Control and Prevention. (2008). Breastfeeding among U.S. children born 1999-2007, CDC national immunization survey. Retrieved from http://www.cdc.gov/breastfeeding/data/NIS_data/ index.htm

9. Callen, J., \& Pinelli, J. (2005). A review of the literature examining the benefits and challenges, incidence and duration, and barriers to breastfeeding in preterm infants. Advances in Neonatal Care, 5, 72-88. doi:10.1016/j.adnc.2004.12.003

10. Furman, L., Minich, N., \& Hack, M. (2002). Correlates of lactation in mothers of very low birth weight infants. Pediatrics, 109, e57. doi:10.1542/peds.109.4.e57

11. Flacking, R., Ewald, U., Nyqvist, K. H., \& Starrin, B. (2006). Trustful bonds: A key to "becoming a mother" and to reciprocal breastfeeding. Stories of mothers of very preterm infants at a neonatal unit. Social Science o Medicine, 62, 70-80. doi:10.1016/j.socscimed.2005.05.026

12. Sandelowski, M. (2000). Whatever happened to qualitative description? Research in Nursing of Health, 23, 334-340. doi:10.1002/1098$240 \mathrm{X}(200008) 23: 4<334::$ AID-NUR9>3.0.CO;2-G

13. Thorne, S., Kirkham, S. R., \& MacDonald-Emes, J. (1997). Interpretive description: A noncategorical qualitative alternative for developing nursing knowledge. Research in Nursing and Health, 20, 169-177. doi:10.1002/ (SICI)1098-240X(199704)20:2<169::AID-NUR9>3.0.CO;2-I_

14. Gatti, L. 2008. Maternal perceptions of insufficient milk supply in breastfeeding. Journal of Nursing Scholarship, 40, 355-363. doi:10.1111/ j.1547-5069.2008.00234.x

15. McCarter-Spaulding, D. E., \& Kearney, M. H. (2001). Parenting selfefficacy and perception of insufficient breast milk. Journal of Obstetric, Gynecologic, and Neonatal Nursing, 30, 515-522. doi:10.1111/j.15526909.2001.tb01571.x

16. Hill, P., Aldag, J., \& Chatterton, R. (2001). Initiation and frequency of pumping and milk production in mothers of nonnursing preterm infants. Journal of Human Lactation, 17, 9-13. doi: $10.1177 / 089033440101700103$

17. Meier, P. P. (2001). Breastfeeding in the special care nursery: Prematures and infants with medical problems. Pediatric Clinics of North America, $48,425-442$.

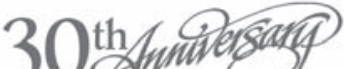


18. Dudek-Shriber, L. (2004). Parent stress in the neonatal intensive care unit and the influence of parent and infant characteristics. The American Journal of Occupational Therapy, 58, 509-520.

19. Miles, M. S., Burchinal, P., Holditch-Davis, D., Brunssen, S., \& Wilson, S. M. (2002). Perceptions of stress, worry, and support in black and white mothers of hospitalized, medically fragile infants. Journal of Pediatric Nursing, 17, 82-88. doi:10.1053/jpdn.2002.124125

20. Flacking, R., Ewald, U., \& Starrin, B. (2007). "I wanted to do a good job": Experiences of "becoming a mother" and breastfeeding in mothers of very preterm infants after discharge from a neonatal unit. Social Science \& Medicine, 64, 2405-2416. doi:10.1016/j.socscimed.2007.03.008

21. Callen, J., Pinelli, J., Atkinson, S., \& Saigal, S. (2005). Qualitative analysis of barriers to breastfeeding in very-low-birthweight infants in the hospital and postdischarge. Advances in Neonatal Care, 5, 93-103. doi:10.1016/j.adnc.2004.12.005

22. Mercer, R. T. (2004). Becoming a mother versus maternal role attainment. Journal of Nursing Scholarship, 36, 226-232. doi:10.1111/ j.1547-5069.2004.04042.x

23. Zabielski, M. T. (1994). Recognition of maternal identity in preterm and fullterm mothers. Maternal-Child Nursing Journal, 22, 2-36.

24. Mizuno, K., Mizuno, N., Shinohara, T., \& Noda, M. (2004). Motherinfant skin-to-skin contact after delivery results in early recognition of own mother's milk odour. Acta Paediatrica, 93, 1640-1645. doi:10.1111/ j.1651-2227.2004.tb00856.x

\begin{abstract}
About the Authors
Camille A. Boucher obtained a research grant from l'agence universitaire de la Francophonie to study the biochemical effects of antioxidants on individuals with sickle cell anemia in Senegal, Africa, in 2004. She completed her bachelor of science degree from the University of Manitoba in 2005. In 2008, she received her master of science in nursing from McGill University. Currently, she is practicing in the pediatric intensive care unit at the Health Science Centre in Winnipeg, Manitoba.
\end{abstract}

Paola M. Brazal received her bachelor of science degree from Queen's University and her master of science in nursing from McGill University. She is currently working as a staff nurse in the emergency department at the Hospital for Sick Children in Toronto, Ontario.

Cynthia Graham-Certosini is a nurse manager at the Jewish General Hospital, faculty member at McGill University, and most recently taught undergraduate students in the clinical setting.

Kathryn Carnaghan-Sherrard is a clinical nurse specialist and consultant in family nursing at the NICU, Jewish General Hospital and a faculty lecturer at the School of Nursing, McGill University.

Nancy Feeley is a nurse researcher at the Jewish General Hospital, faculty member at McGill University, and the codirector of the Quebec Interuniversity Nursing Intervention Research Group.

To the mothers who graciously permitted us to enter their world, providing us with the opportunity to listen to their stories and learn from their personal experiences, thank you. We also wish to express our gratitude to Nancy Vincelli for facilitating our recruitment process and to Kathy Hamelin for her expertise. Without the commitment and devotion of all those involved, this study would not have been possible. Dr. Feeley is supported by a clinical research scholar award from the Quebec Health Research Fund (FRSQ).

For further information, please contact:

Camille A. Boucher, RN, MSc(A)

Health Science Centre

820 Sherbrook Street

Winnipeg, MB R3A 1 R9

Canada

E-mail: camille_boucher@hotmail.com 